\title{
An Overview of Underground Coal Miner Electronic Tracking System Technologies
}

\author{
Carl Sunderman, Joseph Waynert \\ National Institute for Occupational Safety and Health \\ Office of Mine Safety and Health Research \\ Pittsburgh, Pennsylvania, USA \\ cps5@cdc.gov
}

\begin{abstract}
Since the passage of the Mine Improvement and New Emergency Response Act (MINER Act) of 2006, several electronic tracking systems that inform personnel on the surface of a coal mine about the location of miners underground have become commercially available. These systems can be broadly categorized into technology groups that are described in this paper. In addition, several other technologies have been identified by the National Institute for Occupational Safety and Health (NIOSH) Office of Mine Safety and Health Research (OMSHR) that show promise to increase location accuracy, reduce installed infrastructure, or otherwise improve the performance of existing systems. This paper will describe tracking system technologies currently installed in US coal mines and discuss some emerging technologies.
\end{abstract}

Keywords-Miner Tracking Systems; Real Time Locating Systems; Radio Frequency Identification

\section{INTRODUCTION}

Until 2006, personnel tracking in underground coal mines was typically limited to a simple brass tag-in/tag-out system at the portal in conjunction with periodic voice communications over the mine page phone. However, in the summer of 2006, Congress enacted the Mine Improvement and New Emergency Response (MINER) Act [1] in reaction to a series of mine tragedies occurring earlier that year. The MINER Act amended previous mine safety legislation in part by requiring mine managers to submit an emergency response plan to the Mine Safety and Health Administration (MSHA). The plan must provide for an electronic tracking system permitting surface personnel to determine the location of any persons trapped underground [1].A typical room and pillar coal mine is composed of intersecting tunnels called entries and crosscuts spaced relatively uniformly in a checkerboard arrangement, with roof heights between approximately one and three meters, and entry crosscuts spaced every 15 to as much as 30 meters or more. The environment is typically dusty and humid, requiring tracking system components to be environmentally sealed, while the physical hazards involved in mining require that tracking system components be rugged.

To help provide guidance to mine operators in their personnel tracking efforts, MSHA published several Program Policy Letters [2]. According to PPL P11-V-13, tracking systems must determine the location of miners to within 200 feet $(61 \mathrm{~m})$ in working sections or other strategic locations and to within 2000 feet $(610 \mathrm{~m})$ in the primary and secondary escapeways. Locating miners at these accuracies with recently MSHA-approved systems is achievable.

After a disaster, installed tracking systems should ideally survive and remain operational. Tracking systems that have less underground infrastructure are expected to be more survivable; i.e., likely to remain operational after a disaster. Tracking system components must be approved by MSHA as "permissible," requiring either intrinsically safe or explosionproof protection techniques [3]. The reason for this is that mine ventilation may be shut down following an incident leading to increased concentrations of methane in the mine. An electrical spark of sufficient energy can ignite this methane. Intrinsically safe equipment is designed to not produce sparks of sufficient energy to ignite methane, while explosion-proof equipment is designed to not allow explosions to propagate outside of specially designed enclosures. The design constraints from imposing permissibility requirements must be accounted for in developing and producing tracking systems.

At the time of the passage of the MINER Act, no permissible miner tracking systems were available to the industry. The Act established within NIOSH, an Office of Mine Safety and Health, whose purpose was to "enhance the development of new mine safety technology and technical applications and expedite the commercial availability and implementation of such technology in mining environments." [4] Since the passage of the MINER Act OMSHR has supported tracking system development through multiple contract activities and internal research.

Several technologies have been proposed for use in miner tracking systems. Some of these technologies have been incorporated into commercial products while others remain in research and development. NIOSH has published a thorough introduction to miner tracking technologies that are available in commercial products [5]. Additional technologies have also been proposed or are currently being studied. Table 1 outlines all of the technologies known or expected to be applicable in underground coal mines. Table 2 outlines the possible physical configurations that these technologies can be placed into. 
TABLE I

MINER TRACKING SYSTEM TECHNOLOGIES

\begin{tabular}{|l|l|l|l|l|}
\hline Technology & $\begin{array}{l}\text { Tag } \\
\text { cost }\end{array}$ & $\begin{array}{l}\text { Reader } \\
\text { cost }\end{array}$ & Availability & Comments \\
\hline Active RFID & low & med & $\begin{array}{l}\text { Commercially } \\
\text { available }\end{array}$ & $\begin{array}{l}\text { Position only } \\
\text { known near } \\
\text { reader }\end{array}$ \\
\hline $\begin{array}{l}\text { LAN, } \\
\text { PAN }\end{array}$ & med & high & $\begin{array}{l}\text { Commercially } \\
\text { available }\end{array}$ & $\begin{array}{l}\text { Integrated with } \\
\text { communication } \\
\text { system }\end{array}$ \\
\hline $\begin{array}{l}\text { Passive } \\
\text { RFID }\end{array}$ & $\begin{array}{l}\text { very } \\
\text { low }\end{array}$ & med & $\begin{array}{l}\text { Research and } \\
\text { Development }\end{array}$ & $\begin{array}{l}\text { Reliable read } \\
\text { range is short }\end{array}$ \\
\hline $\begin{array}{l}\text { Inertial } \\
\text { MEMS }\end{array}$ & high & $\begin{array}{l}\text { none- } \\
\text { high }\end{array}$ & $\begin{array}{l}\text { Research and } \\
\text { Development }\end{array}$ & $\begin{array}{l}\text { Ideally could } \\
\text { operate } \\
\text { standalone. }\end{array}$ \\
\hline UWB & $\begin{array}{l}\text { low- } \\
\text { med }\end{array}$ & $\begin{array}{l}\text { med- } \\
\text { high }\end{array}$ & $\begin{array}{l}\text { Research and } \\
\text { Development }\end{array}$ & $\begin{array}{l}\text { Requires tight } \\
\text { time } \\
\text { synchronization. }\end{array}$ \\
\hline NFC & low & high & $\begin{array}{l}\text { Research and } \\
\text { Development }\end{array}$ & $\begin{array}{l}\text { Good multipath } \\
\text { performance }\end{array}$ \\
\hline
\end{tabular}

There are currently three configurations of permissible tracking systems commercially available: "zone-based" active Radio Frequency Identification (RFID), "reverse" active RFID, and "radio node"-based. Table 2 summarizes these configurations.

TABLE II

TRACKING SYSTEM CONFIGURATIONS

\begin{tabular}{|l|l|l|l|l|}
\hline Configuration & Accuracy & Survivability & Cost & Comments \\
\hline $\begin{array}{l}\text { Zone-based } \\
\text { RFID }\end{array}$ & low & low & low & $\begin{array}{l}\text { Position only } \\
\text { known near } \\
\text { reader }\end{array}$ \\
\hline Reverse RFID & low-high & high & med & $\begin{array}{l}\text { A system based } \\
\text { on UWB has } \\
\text { high accuracy }\end{array}$ \\
\hline $\begin{array}{l}\text { Radio Node- } \\
\text { based }\end{array}$ & $\begin{array}{l}\text { med- } \\
\text { high }\end{array}$ & low & high & $\begin{array}{l}\text { Integrated with } \\
\text { communication } \\
\text { system }\end{array}$ \\
\hline
\end{tabular}

The zone-based active RFID configuration is shown in Figure 1. Systems based on this configuration are typically implemented with active RFID components. These components are small, miner-worn, battery-powered "tags," each containing a unique identifier, and more complex tag "readers." Tag readers are typically line-powered, networked, infrastructure devices that are used to retrieve the identifiers from nearby tags. In Figure 1, mobile tags are represented with green circles and fixed readers with red circles. Typically there are two different ways that the readers relay messages up to the mine operations center (MOC). The first way is by a dedicated reader network shown as a solid red line in the figure. The second way is by relaying messages over to the primary communication system of the mine shown as a dashed red line in the figure.

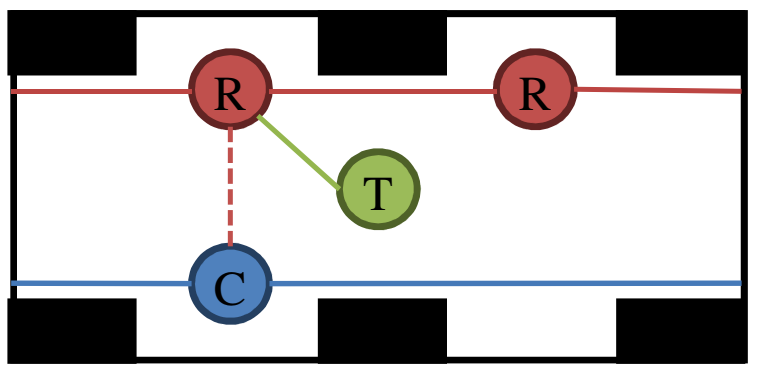

Figure 1: Zone Based RFID System. (Tags in green, readers in red, primary communication system in blue)

A "reverse" RFID configuration is formed when the location of tags and readers are interchanged. Tags, now fixed on the mine infrastructure, contain the known location within the mine, while the readers are made mobile (miner worn). Readers typically relay position information to the primary communication system. Figure 2 shows this configuration.

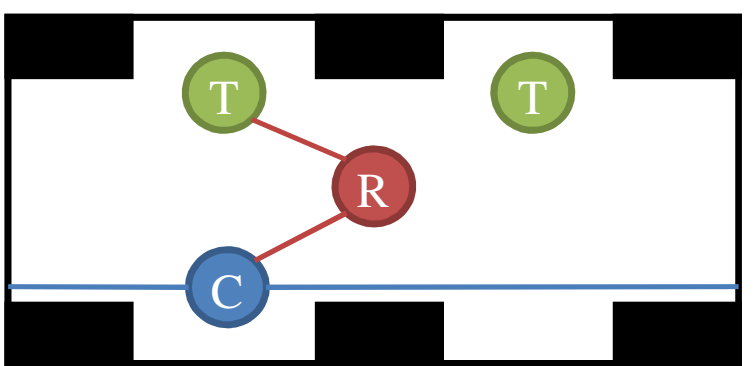

Figure 2: Reverse RFID System. (Tags in green, readers in red, primary communication system in blue)

Figure 3 shows the radio node-based configuration. In this configuration the mine's communication system doubles as a tracking system. Components typically consist of wireless access points (nodes) and handheld radios. The handheld radio periodically connects with nearby nodes to indicate its presence. Nodes can be separated by up to $600 \mathrm{~m}$ and still provide continuous communication coverage, depending on the manufacturer and mine specific considerations. Position is often determined with received signal strength indicator (RSSI) or time difference of arrival (TDOA) methods. RSSI is the amount of RF power contained in the transmission and sensed by the receiver. The falloff in RF power versus distance can be estimated for a given mine environment. Comparing this relationship to the RSSI value yields an estimate of the transmitter to receiver spacing. TDOA is the process of computing the difference in the arrival times of a pulse at two or more nodes, yielding the relative separation of the radio from each node. Tracking information is relayed to the MOC along with other forms of communication. 


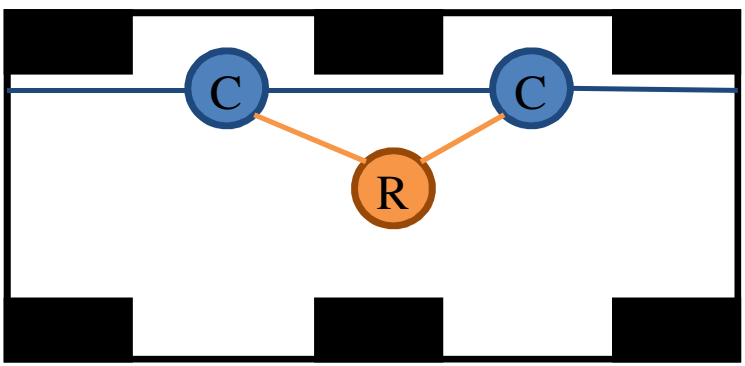

Figure 3: Node-based System. (Radios in orange, primary communication system nodes in blue)

\section{CURRENTLY INSTALLED TECHNOLOGIES}

At the time of this writing, tracking systems installed in US coal mines employ zone-based active RFID, node-based local area networks (LAN), or node-based personal area network (PAN) technologies [6].

Zone-based active RFID based systems are sometimes standards based and other times proprietary. When standards based they often conform to the ISO (International Organization for Standardization) 18000 standard. ISO 18000 is a set of standards describing the behavior of a diverse set of RFID technologies [7]. In both standards based and proprietary systems, tags are typically short-read range devices and communicate with readers when in close proximity to them. The tags are specifically designed to transmit limited information in addition to the identifier, although some manufacturers have incorporated a text transceiver in their readers. However, for simple tags, reliability is ensured, cost is low, and battery life of the tag can be years.

Node-based local area networks are typically implemented with IEEE 802.11 (Wi-Fi) [8]. Wi-Fi is versatile. Many system components can communicate with Wi-Fi nodes in the mine. These can include Voice over Internet Protocol (VOIP) phones, mine automation equipment, and tracking tags. Small lightweight tracking tags can periodically "wake up," associate with a Wi-Fi node, transmit an identifier, and go back into a low power state thus saving battery power. Alternatively, a miner's handheld radio can include the tracking function. Some systems utilize RSSI to increase additional resolution. Nodes are typically line powered but contain battery backup for the event of loss of AC power. Nodes can be interconnected in hardwired, fixed wireless, or wireless mesh configurations. Refer to [1] for a description of Wi-Fi communication systems.

Node-based personal area networks are typically implemented with IEEE 802.15 [9]. Both high and low data rate forms of IEEE 802.15 are installed in mines.

High data rate personal area network devices based on IEEE 802.15 can be used similarly to local area network systems; however, the number of available communication channels is reduced due to the structure of the protocol. Both voice and data communications are supported with handheld radios. Tracking functionality is also built into the systems.
Low data rate personal area network-based tracking systems often use Zigbee (IEEE 802.15.4). In this technology all system components, readers, and tags communicate wirelessly in a mesh network. In a mesh network, reader associations are made automatically so that a message from any tag has a path to the surface. The system is self-healing such that if readers fail, messages can find a way around the failure. Systems are composed of low-cost tags of various forms and readers that receive transmitted tag data. Tag batteries typically can last for years depending on data transmission intervals. Readers are typically line powered but contain battery backup for the event of loss of AC power. Due to the low power requirements, temporary battery-powered readers are available from some suppliers. Advantages of this system over Wi-Fi include dramatically lower power requirements and simpler network protocol. However, the lower power requirements of Zigbee systems limit the available bandwidth for other forms of communication. This is the main disadvantage of the system as it currently does not support voice communication. Currently implemented systems are typically limited to twoway text communication in addition to the tracking function.

\section{EMERGING TECHNOLOGIES}

Since the passage of the MINER Act, several alternative technologies have been either investigated by or demonstrated to OMSHR. These technologies include passive RFID, ultrawideband, inertial sensing, and near field ranging.

Passive RFID technology is an attractive alternative to active RFID in that passive tags do not contain a battery. Instead they store received RF energy while interrogated and use it to power (or backscatter) their identifier. Compared with active RFID tags, initial cost is lower and batteries do not need to be periodically replaced. However, read range is reduced and reception can be less reliable [10]. New, compact, and sensitive readers have recently become available. Also, the performance of tags has been improving to the point where a tracking system based on passive RFID technology may become feasible, however, up to the present, reliable readings have not been demonstrated.

Zone-based passive RFID systems are commonplace in asset control and warehousing applications. A reliable system based on a zone configuration may be achievable in the future based on RFID device-level improvements [10].

A "reverse passive" RFID system has also recently been proposed. In this configuration the tags are fixed on the mine infrastructure and the reader is carried by the miner. Tags, due to their low cost and rugged nature, can be installed uniformly throughout the mine. The mobile reader, carried by the miner, computes its local position from the pre-surveyed nearest neighbor tags. The position estimate is then relayed to the surface over the primary communication system of the mine. The difficulty with this approach is accounting for the possibility of multiple readers operating in close proximity to one another at the same time. 
Another promising active RFID technology is ultrawideband (UWB) ranging. Coal mines exhibit significant multipath fading effects to the propagation of narrowband signals [1]. The multipath fading effect occurs when waves from the direct path interact with waves from reflected paths (e.g. off of mine tunnel walls). At some locations along the tunnel the waves add (or intensify) and at other locations the waves subtract (or nullify). These effects could introduce unacceptable errors to the location algorithm. UWB signals solve this problem by dramatically spreading the radio spectrum, reducing the effect of multipath fading. Current reductions in the cost and size of UWB radios make this technology particularly attractive.

A system based upon a "reverse" UWB implementation has also been developed. As with the reverse passive RFID technology discussed above, the tags become part of the infrastructure of the mine, attached to the roof. The UWB reader is carried by the miner and powered by the cap lamp battery. The reader listens to the periodic transmissions from the UWB tags and computes its position based on the RSSI values of the received data. The system then relays the position estimates to a primary communication system with a bridge device. This system has been shown, with tags spaced approximately $60 \mathrm{~m}$ apart, to have an accuracy of 12 meters [11].

A "forward" UWB implementation may also be possible. The idea is that instead of measuring RSSI, readers sense the arrival time of a short pulse from a tag. Computing the difference in the arrival times of a pulse at two or more readers can yield the separation distance of the tag from each reader. This technique is called differential time of arrival (DTOA). DTOA also presents difficulties: In order to accurately measure differences in time at each reader the clock on all readers must be tightly synchronized. In the event of a disaster the lack of synchronization would lead to dramatically increased location error with time.

Dead reckoning based on inertial measurement units (IMU) is another alternative technology. An IMU based on microelectro-mechanical systems (MEMS) accelerometers and gyroscopes estimates position of an object based solely upon its movements. Theoretically, in the absence of noise, the device could reckon its position without other external input. This would be desirable since tracking system infrastructure components could be eliminated. In practice, however, due to drift caused by intrinsic noise in the MEMS sensor elements and misalignment errors, even the most accurate MEMS IMUs can maintain reasonable accuracy for only a minute or less [12]. These errors are principally caused by a double mathematical integration of the acceleration values sensed by the MEMS accelerometers. An IMU thus must be corrected by some other known reference relatively often; otherwise the intrinsic drift will compromise accuracy. Normally these corrections would come from tracking system infrastructure components such as readers. Drift and cost of MEMS IMU sensors are continually decreasing, potentially making these sensors applicable to miner tracking systems in the future; however, current inexpensive devices appear to be inadequate.
Near field communication (NFC) ranging technology has been demonstrated in a test mine. In this solution, medium frequency (around 1 megahertz) transmissions are used to estimate position. A fixed transmitter broadcasts a signal to mobile receivers. When the distances between transmitter and receiver are less than the wavelength (around 300 meters) then the electromagnetic energy is in the near field. When in the near field the electric and magnetic field components of the transmission decrease from 90 degrees to 0 degrees as a receiver moves away from the transmitter into the far field (distances greater than a wavelength). A receiver that measures these phase differences can compute an estimate of position from the transmitter. In one demonstration, with transmitting node separations of up to 70-m intervals, less than 1-m resolution was obtained [13].

\section{CONCLUSION}

Tracking system technologies play an important role in the safety of America's coal miners. The ability for mine rescue teams to know the approximate location of trapped victims will speed the recovery effort in the event of a disaster. Current technologies, based on zone- and node-based systems, are adequate to satisfy MSHA guidance on minimum resolution. Emerging technologies show promise to reduce cost and increase positional resolution. In addition, some developing technologies require less underground infrastructure which could make the systems more survivable. There is still much to be learned about tracking systems as mines gain operational experience over the next few years.

\section{DISCLAIMER}

The findings and conclusions in this report are those of the authors and do not necessarily represent the views of NIOSH.

\section{REFERENCES}

[1] T Novak, D Snyder and J Kohler. "Post-accident Mine Communications and Tracking Systems," IEEE Transactions on Industry Applications, Vol 46, No 2. March/April 2010.

[2] Mine Safety and Health Administration, "Program Policy Letter No. P11-V-13,” April 28, 2011.

[3] US Code of Federal Regulations, 30CFR18.

[4] United States Public Laws, PL 109-236, Mine Improvement and New Emergency Response Act of 2006 (MINER Act), Jun. 15, 2006.

[5] DHHS (NIOSH) Tutorial on Wireless Communication and Electronic Tracking, Part 2: Advanced.

[6] D Chirdon, MSHA, Personal communication, February 2012.

[7] ISO/IEC 18000-1:2008 Information technology—Radio frequency identification for item management-Part 1: 
Reference architecture and definition of parameters to be standardized.

[8] IEEE 802.11: Wireless LAN Medium Access Control (MAC) and Physical Layer (PHY) Specifications. (2007 revision).

[9] IEEE Standard 802.15.1-2005-Part 15.1: Wireless Medium Access Control (MAC) and Physical Layer (PHY) Specifications for Wireless Personal Area Networks (WPANs).

[10] RFID Journal, "How to Choose the Right RFID System: A Step by Step Guide", RFID Journal Special Report, 2011.

[11] DHHS (NIOSH) Publication No. 2011-209, TN 543, 2011 Sept.

[12] O Woodman. "An introduction to inertial navigation." Technical Report 696, University of Cambridge, 2007.

[13] H Schantz, "A real-time location system using near-field electromagnetic ranging," 2007 IEEE Antennas and Propagation International Symposium, 9-15 June, 2007

"U.S. Government work not protected by U.S. copyright" 